\title{
Resistance of Some Bacteria Isolated From Urinary Tract Infections in Elderly Patients to Antibiotics and Detection of $\beta$-lactamases in Them
}

\author{
Mohammed Abdullah Mahmood ${ }^{1}$, Shaker Gazi Gergees ${ }^{2}$ Alaa Taha Younis ${ }^{3}$, \\ ${ }^{1,3}$ Department of Biology, College of Education for Pure Science, University of Mosul, Iraq. \\ ${ }^{2}$ Department of Biology, College of Sciences, University of Mosul, Iraq
}

E-mail: ${ }^{1}$ mohammedalmola1981@uomosul.edu.iq , ${ }^{2}$ momo12345mola@yahoo.com, ${ }^{3}$ Alaatyounis@uomosul.edu.iq

(Received May 15, 2021; Accepted June 16, 2021; Available online August 28, 2021)

DOI: 10.33899/edusj.2021.168647, @ 2021, College of Education for Pure Science, University of Mosul.

This is an open access article under the CC BY 4.0 license (http://creativecommons.org/licenses/by/4.0/).

\begin{abstract}
Urinary tract infection is the second-largest infection, especially in elderly people, and the increasing use of antibiotics has led to the emergence and development of resistance in isolated and other bacteria in this study Fifty urine samples from elderly patients with urinary tract infections were collected from both sexes in Mosul city. Twenty-six bacterial isolates were isolated and diagnosed by ordinary bacteriological tests. The isolates were related to the species. Escherichia coli, Klebsiella pneumoniae, Pseudomonas aeruginosa and Proteus mirabilis. After isolation, their resistance to 14 selected antimicrobial agents was tested by the disk diffusion method. The presence of $\beta$-lactamases, in general, was detected by acidimetric method, the detection of extended-spectrum $\beta$-lactamases(ESBLs) was performed by double disk method, while the detection of AmpC $\beta$ lactamases was performed by cefoxitin resistance method. The isolates showed clear resistance to $\beta$-lactam antibiotics ranged (50\%--92.3\%), and to ciprofloxacin, gentamicin, sulfamethoxazole, streptomycin, rifampin with rates; $(53.8 \%),(57.7 \%),(61.5 \%),(80.8 \%),(88.5 \%)$, respectively, while they showed reduced resistance to amikacin (15.4\%). $\beta$-lactamases in general were detected in $(96.1 \%)$ of the isolates, while $(19.2 \%)$ of them gave a positive result for the detection of (ESBLs). AmpC $\beta$-lactamases were detected in $(69.2 \%)$ of the isolates as they showed a clear resistance to cefoxitin which indicates that they are potential producers of these enzymes.
\end{abstract}

Keywords: Pathogenic bacteria, $\beta$-lactamases, antibiotics

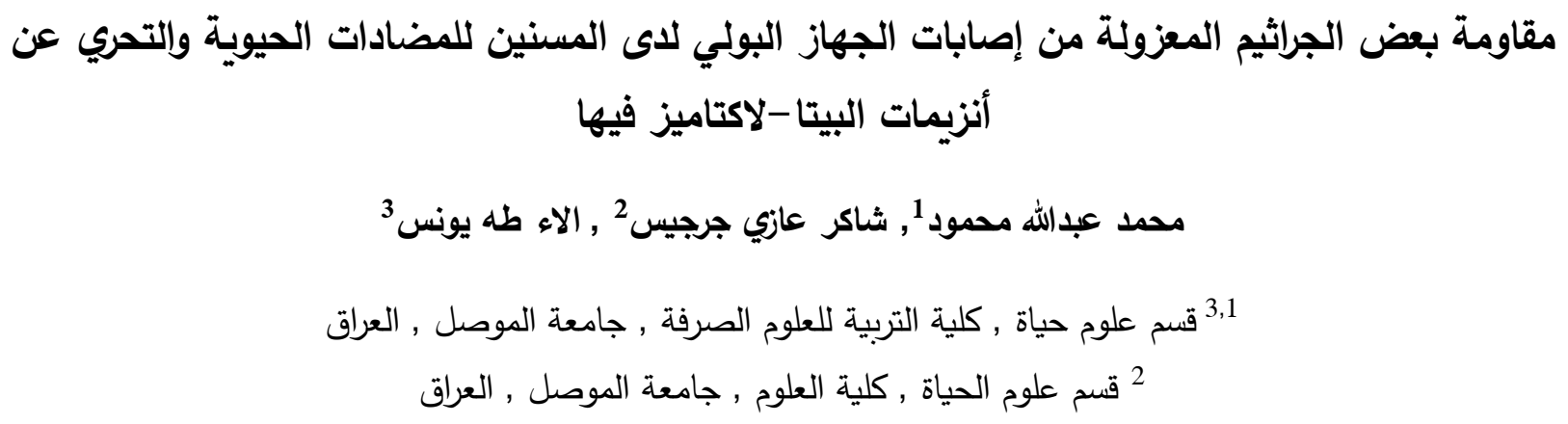


عدوى المجاري البولية ثاني اكبر عدوى شيوعا وخاصـة في الاثخاص المسنين و الاستخدام المتزايد للمضـادات الحيوية ادى الى ظهور وتطور المقاومة لاى الانواع البكتريا المعزولة ,في هذه الدراسة جمعت 50 عينة ادرار من إصابات الجهاز البولي

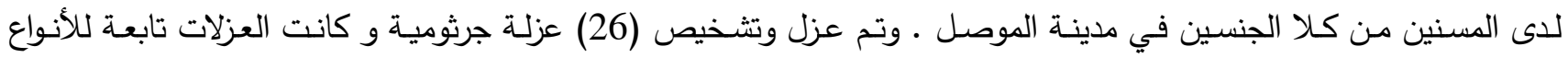
Proteus mirabilis، Pseudomonas aeruginosa، Klebsiella pneumoniae ، Escherichia coli مقاومتها لـ(14) نوعاً من المضـادات الحيويـة بطريقة الانتثار بالأقراص ـ تم التحري عن تواجد كلا من أنزيمات البيتا-لاكتاميز

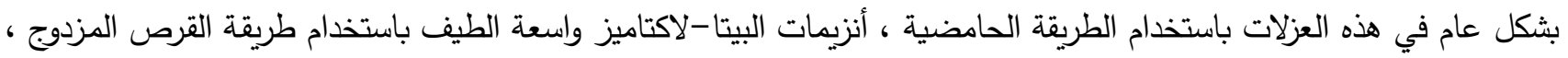

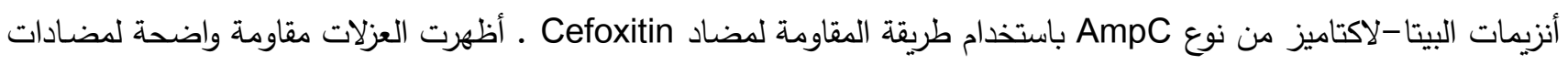

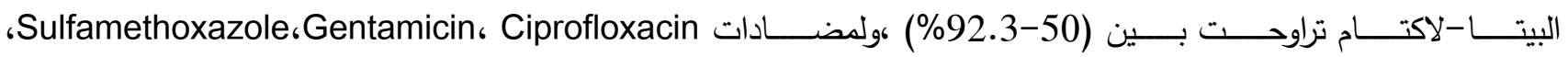
Rifampin ، Streptomycin

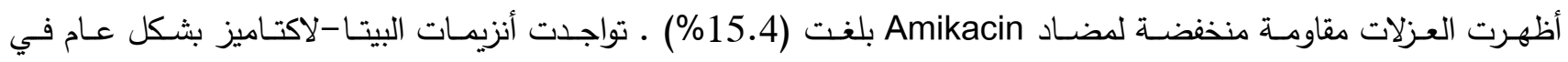

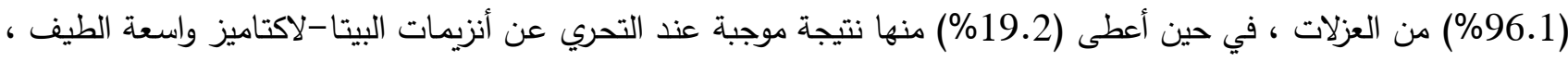

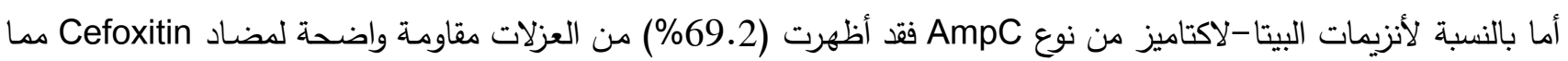

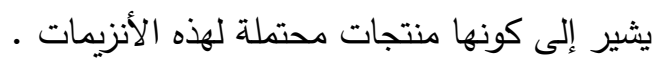

الكلمات المفتاحية: الجراثيم المرضية, انزيمات البيتا-لاكتاميز ، المضادات الحيوية

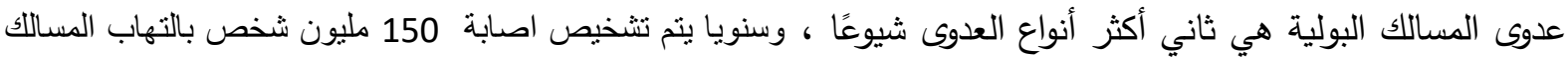
البولية في جميع أنحاء العالم ، مما يكلف الاقتصاد العالمي أكثر من 6 مليار دولار (1). إن مسببات عدوى المسالك البولية يمكن

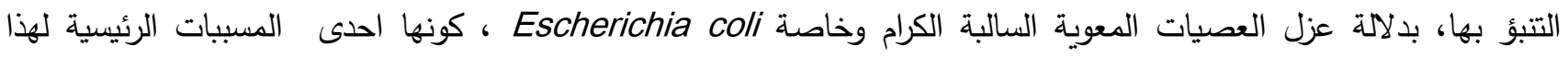
المرض (2). يتم التعامل مع التهاب المجاري البولية الأكثر شيوعًا للاغراض العلاجية باستخدام عوامل مضادة للميكروبات الاتي

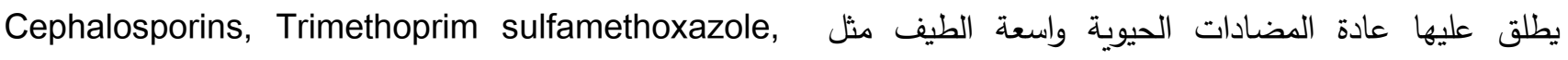
Penicillins

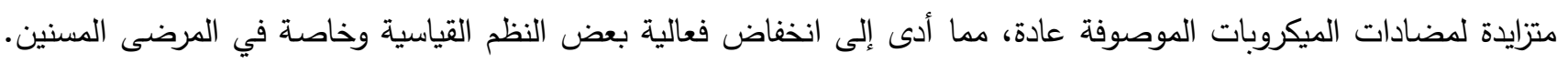

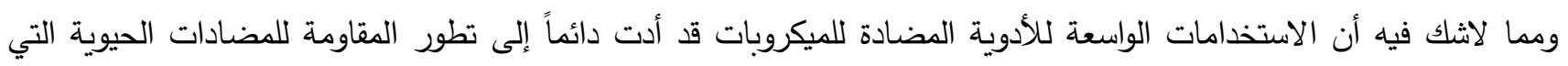
أصبحت في السنوات الأخيرة مشكلة رئيسية في جميع أنحاء العالم (4). تعد أنزيمات البيتا-لاكتاميز lactamase - م من أهم الاليات التي تستخدمها البكتريا في مقاومة مضادات البيتا -لاكتام ، عن

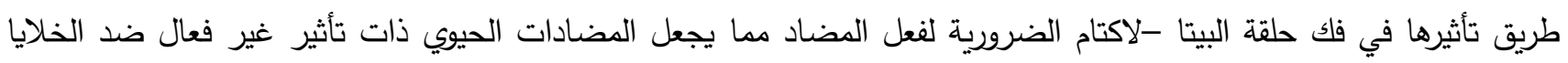

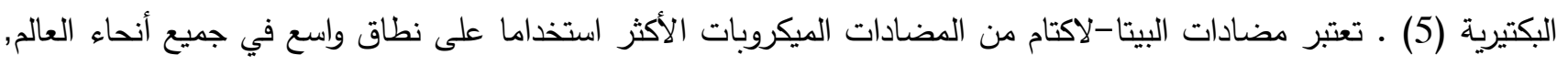

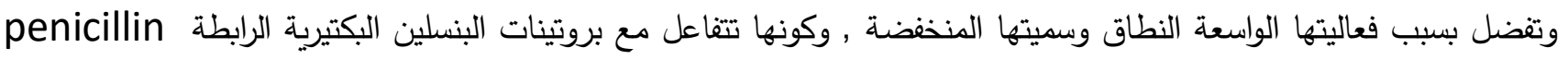
(PBPs) binding proteins

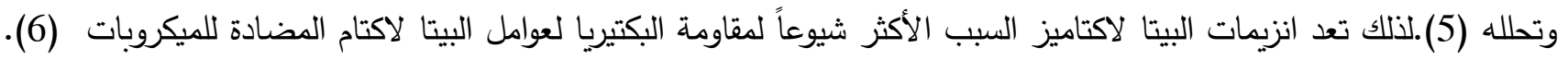
لقد تم تسجل ما يزيد عن (250) نوعا من هذه الانزيمات، كما وضعت تصانيف خاصة بها من اجل تسهيل تثخيصها وتحديد 
أنواعها نظرا لتزايد أعدادها بشكل مستمر وقد صنفت في مجموعات أو فئات من الطيف المائي، والتعرض للمثبطات وما إذا كانت مشفرة من قبل كروموسوم أو البلازميدات (7).

وتتميز أنزيمات البيتا -لاكتاميز واسعة الطيف ( ESBLs) بتحليلها لعدد كبير من مضادات البيتا-لاكتام مثل البنسلينات واسعة الطيف والسيفالوسبورينات الاولى وكذلك سيفالوسبورينات الجيل الثالث، ومما يميز هذه ألانزيمات تثبيطها بحامض الكالفيولين Clavulinic acid methoxy-cephalosporins (7)TEM انزيمات البيتا لاكتاميز تتشأ من خلال الطفرات التي تأثر على إلى واحد أو أكثر من بدائل الأحماض الأمينية التي تغير التكوين

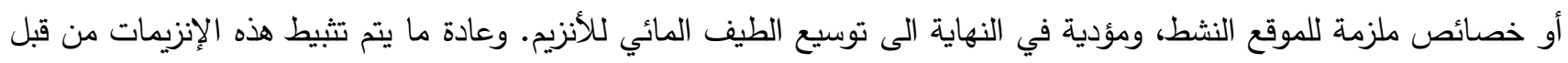
مثبطات البيتا لاكتاميز مثل (tazobactam, sulbactam ،clavulanic acid). (8,10,11)

ومن امثلت هذه الأنزيمات انزيمات البيتا-لاكتاميز من نوع AmpC تتميز بتحليها للعديد من مضادات البيتا -لاكتام بما فيها

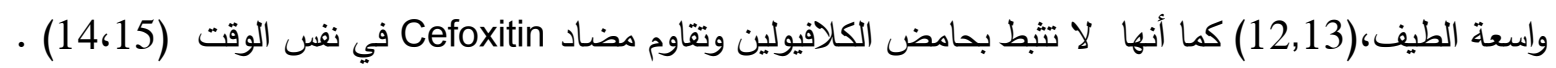

في هذه الدراسة تم الكثف عن حساسية العصيات البولية السالبة الكرام المعزولة للمضادات الميكروبات وأنماط المقاومة في مدينة

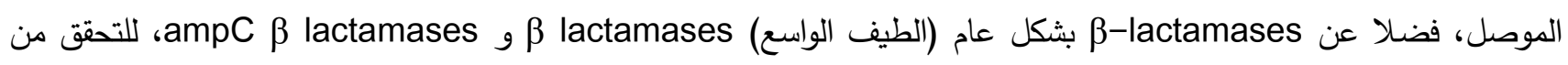
جزء من الوضع الحالي لمقاومة المضادات الحيوية في العراق.

المواد وطرائق العمل العزل والتشخيص

أجريت الدراسة على المرضى المسنين الذين تتراوح أعمارهم بين (50-80 سنة) المصابين بالتهابات المسالك البولية الذين راجعوا مستثفى ابن سينا التعليمي في مدينة الموصل / العراق خلا فترة ثلاثة أشهر تم جمع عينات الادرار التي تم الحصول

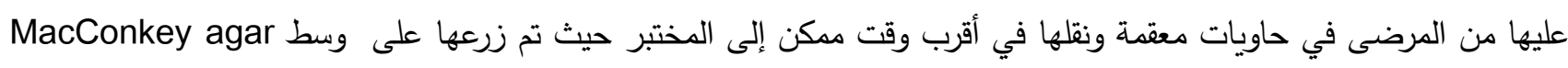

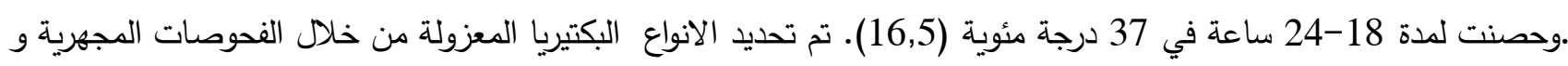

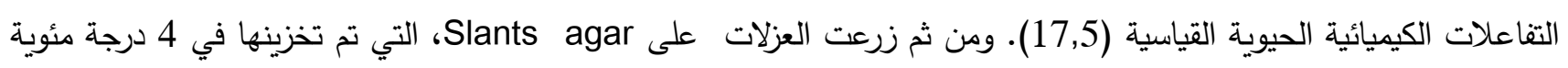
لحين اجراء التجارب اللاحقة عليها . التحري عن الانزيمات الواسعة الطيف

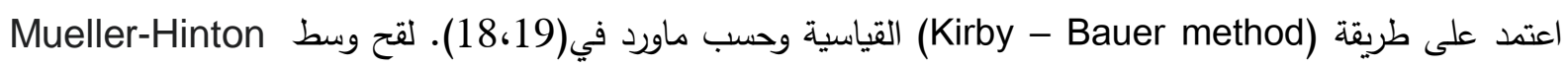

agar بالمعلق الفتي من البكتريا باستخدام ماسحة قطنية معقدة تم بعدها توزيع أقراص المضادات التالية (Amoxicillin25رو) ,

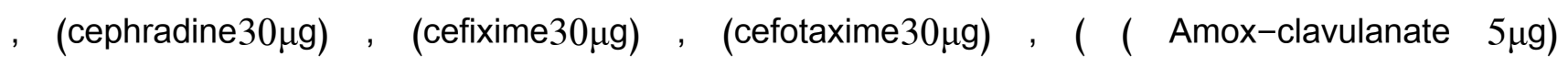

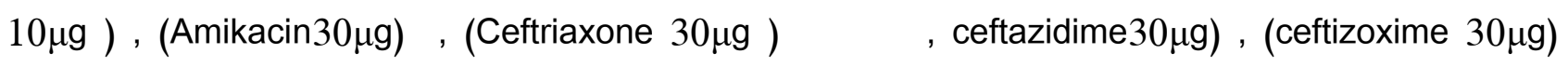
(sulfamethoxazole100 $\mu \mathrm{g})($ Rifampin $5 \mu \mathrm{g}), \quad($ Gentamicin $10 \mu \mathrm{g})$ , (Streptomycin

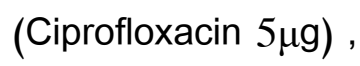


التي تم الحصول عليها من شركة Bioanalyse Co.، تركيا. والمعايير التفسيرية لكل مضادات الميكروبات هي تلك التي أوصت بها اللجنة الوطنية للأمراض والأمراض المعدية (20).

التحري عن انزيم البيتا-لاكتاميز باستخدام الطريقة الحامضية

تم التحري عن وجود lactamases-la بطريقة قياس الحموضة (21). تم تخفيف 2مل من 0.5\% محلول أحمر الفينول

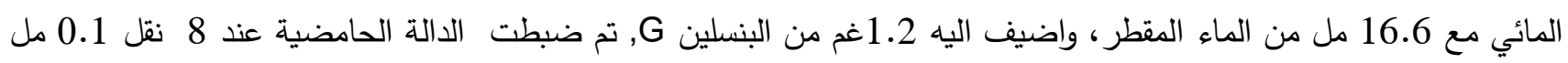
من الكاشف الى انبوبة اختبار ولقحت بعد ذلك بالبكتريا النامية في الوسط الصلب لانتاج معلق كثيف تركت الانابيب بدرجة حرارة الغرفة وتم مراقبة تغير اللون. علما ان ظهور اللون الاصفر خلال 5 دقائق يعد نتيجة موجبة للاختبار (11). الكثف عن ESBLs تأزر القرص المزدوج

لقح وسط أكار مولر -هنتون بالمعلق الفتي من البكتيريا باستخدام ماسحة قطنية معقمة وحسب توصيات اللجنة الوطنية

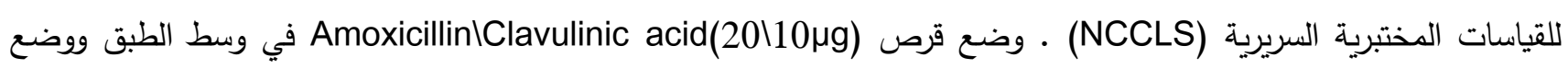

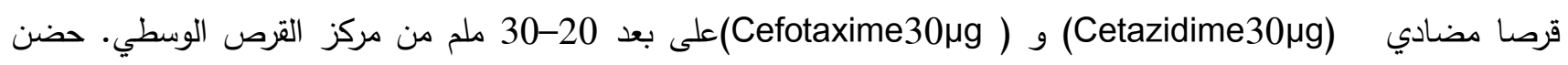
الطبق بدرجة حرارة30 مْ لمدة 24 ساعة ، تم بعدها ملاحظة مدى وجود أو غياب اتساع منطقة التثبيط في احدى أو في كلا المضادين باتجاه القرص الوسطي $(22,23$ (24)

التحري عن انزيمات AmpC المستحثة

تم التحري عن هذه الانزيمات في البكتريا بشكل اولي اعتمادا على مقاومتها للمضاد Cefoxitin بتركيز 30 مايكروغرام /قرص وبالتالي فإن مقاومة Cefoxitin يمكن أن تستخدم كمؤشر رئيسي على وجود هذه الانزيمات في العزلات المشتبه بها (25).

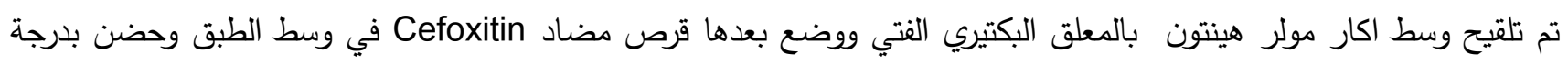

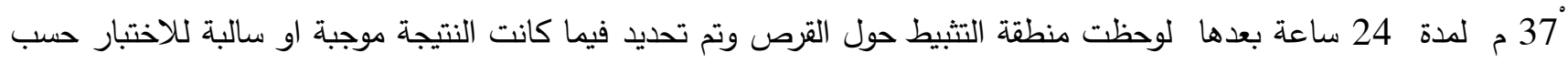
قياسات

النتائج والمناقشة

تم جمع50 عينة ادرار من المرضى المسنين (28 أنثى و 22 من الذكور) تتراوح أعمارهم بين 50 و 80 سنة.اظهرت

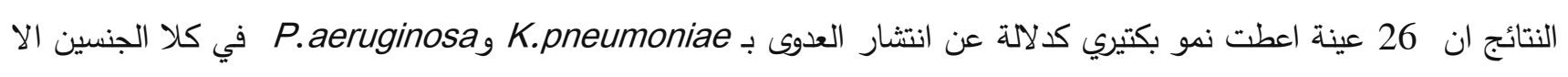
انها كانت أكثر انتشارا في الإناث، في حين أن العدوى E.coli و Pr.mirabilis وزعت بالتساوي تقريبا بين الجنسين (الجدول 1)،

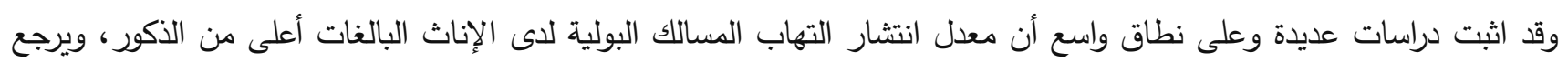
ذلك أساسا إلى عوامل تشريحية وبدنية (4). 
الجدول (1) عدد العزلات الجرثومية والنسبة المئوية للمرضى في كلا الجنسين

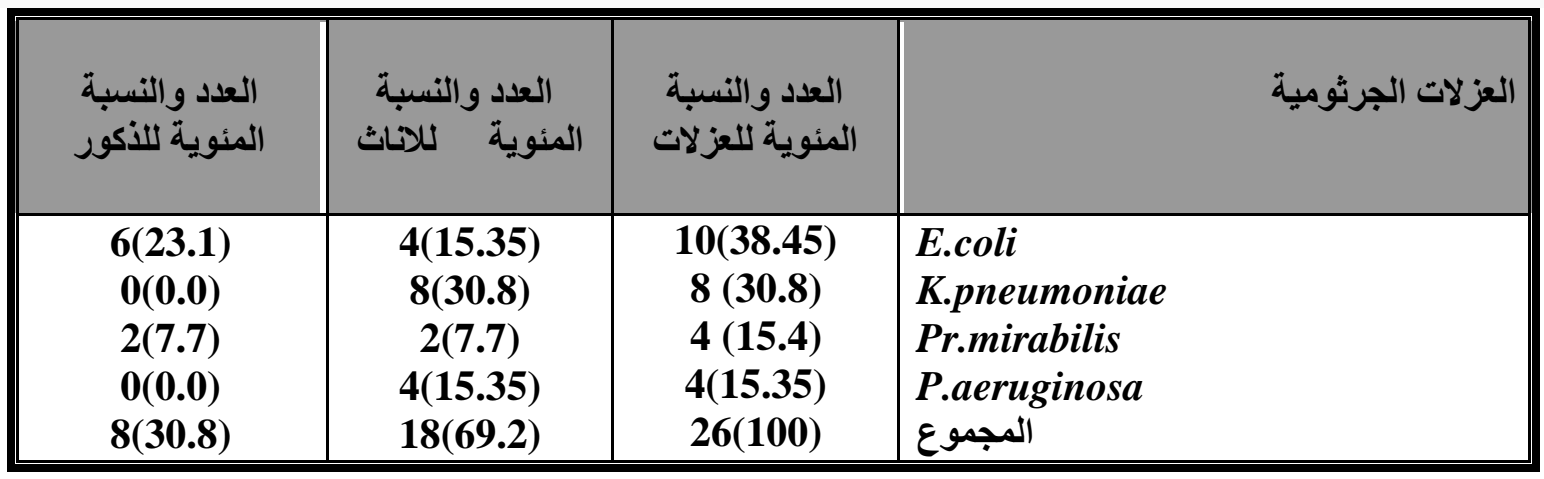

يبين الجدول (2) ملخصاً لفاعلية وطيف 14 نوع من المضادات على البكتريا المعزولة ـ أظهرت العزلات معدلات مقاومة

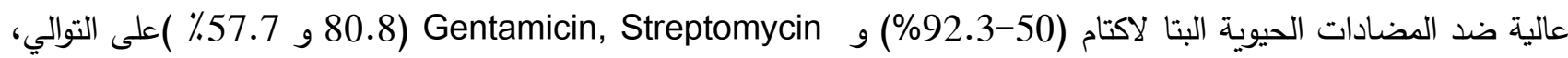

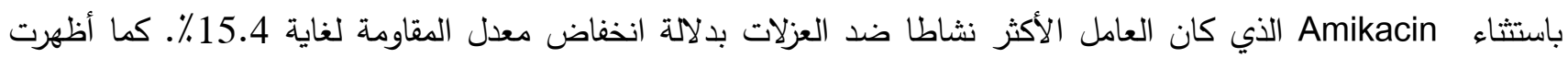

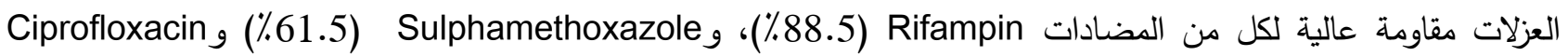
(53.8٪). وبالاضافة لذلك، وجد أن جميع العزلات التي تضمنتها الدراسة تقريباً مقاومة لأربعة أو أكثر من المضادات الحيوية ولوحظ أربعة وعشرون نمط من أنماط المقاومة المختلفة بين العزلات. تم العثور على كل من هذه الأنماط في عزلة واحد، وتكرر نمطين فقط في عزلتي

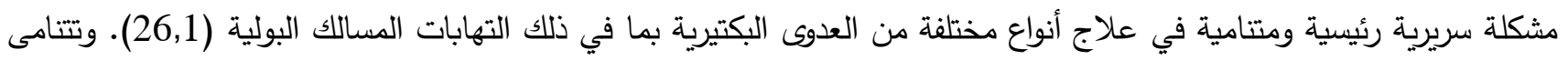

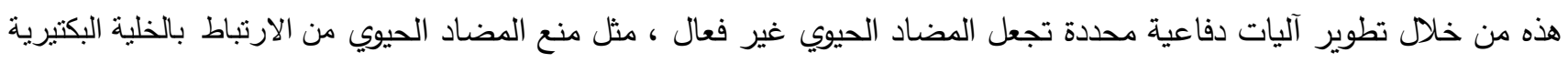
او منعه من دخول الخلية او ضخه ، وإنتاج الإنزيمات التي تعطل المضادات الحيوية (البيتا لاكتاميز، والأمينوكليكوسيدات المعدلة للإنزيمات)، وتغيير مواقع الارتباط الداخلي (الهدف) للمضادات الحيوية تغيير في PBPs أو البروتينات الريبوسومية (27,25,5) المضادات الحيوية بين مسببات الأمراض البكتيرية (15,8) 


$$
\text { الجدول(2) النسبة المئوية لمقاومة البكتريا لعدة انواع من المضادات الحيوية }
$$

\begin{tabular}{|c|c|c|c|c|c|}
\hline $\begin{array}{c}\text { E.coli_} \\
\text { العدد و(\%) }\end{array}$ & $\begin{array}{c}\text { K.pneumoniae } \\
\text { العدد و\%(\% }\end{array}$ & $\begin{array}{l}\text { Pr.mirabilis_ } \\
\text { العدد و(\%) }\end{array}$ & $\begin{array}{l}\text { P.aeruginosa } \\
\text { العدد و(\%) }\end{array}$ & والعد الكلي & المضادات الحيوية \\
\hline $10(100)$ & $8(100)$ & $2(50)$ & $4(100)$ & $24(92.3)$ & Cephradine \\
\hline $9(90)$ & $8(100)$ & $2(50)$ & $4(100)$ & $23(88.5)$ & Amoxicillin \\
\hline $9(90)$ & $8(100)$ & $2(50)$ & $4(100)$ & $23(88.5)$ & Rifampin \\
\hline $8(80)$ & $8(100)$ & $2(50)$ & $4(100)$ & $22(84.6)$ & Amox-clavulanate \\
\hline $8(80)$ & $7(87.5)$ & $3(75)$ & $4(100)$ & $22(84.6)$ & Cefixime \\
\hline $8(80)$ & $7(87.5)$ & $2(50)$ & $4(100)$ & $21(80.8)$ & Streptomycin \\
\hline $7(70)$ & $7(87.5)$ & $2(50)$ & $4(100)$ & $20(76.9)$ & Ceftizoxime \\
\hline $6(60)$ & $7(87.5)$ & $1(25)$ & $2(50)$ & $16(61.5)$ & Cefotaxime \\
\hline $5(50)$ & $6(75)$ & $3(75)$ & $2(50)$ & $16(61.5)$ & Sulphamethoxazole \\
\hline $4(40)$ & $8(100)$ & $\mathbf{0}(\mathbf{0 . 0})$ & $3(75)$ & $15(57.7)$ & Gentamicin \\
\hline $5(50)$ & $7(87.5)$ & $1(25)$ & $2(50)$ & $15(57.7)$ & Ceftriaxone \\
\hline $5(50)$ & $6(75)$ & $1(25)$ & $2(50)$ & $14(53.8)$ & Ciprofloxacin \\
\hline $4(40)$ & $6(75)$ & $1(25)$ & $2(50)$ & $13(50)$ & Ceftazidime \\
\hline $2(20)$ & $2(25)$ & $\mathbf{0}(\mathbf{0 . 0})$ & $\mathbf{0}(\mathbf{0 . 0})$ & $4(15.4)$ & Amikacin \\
\hline
\end{tabular}


Journal of Education and Science (ISSN 1812-125X), Vol: 30, No: 3, 2021 (198-208)

Special Issue for Proceeding of $3^{\text {rd }}$ National (1st international conference of biology) (ICBSUM 2021) 5, 6 July College of Education for Pure Science, University of Mosul, Mosul, Iraq.

$$
\text { الجدول(3) انماط مقاومة المضادات الحيوية للبكتيريا المعزولة في الدراسة }
$$

\begin{tabular}{|c|c|c|}
\hline انماط المقاومة & عدد العزلات & الأواع الجرثُمية \\
\hline 1- مقاومة لجميع المضادات ماعدا CAZ & 1 & E.coli \\
\hline 2- مقاومة لجميع المضادات ماعدا AK & 1 & \\
\hline 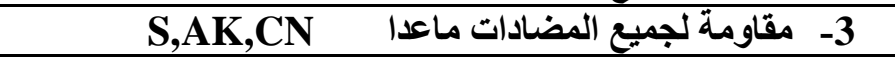 & 1 & \\
\hline 4- مقاومة لجميع المضادات ماعدا SMZ,AK,CN & 1 & \\
\hline $\mathrm{Cep}^{3}, \mathrm{AMC}, \mathrm{RA}, \mathrm{AX}, \mathrm{CE}, \mathrm{CIP}-5$ & $\mathbf{1}$ & \\
\hline AMC,CE,CTX,ZOX,CFM,RA,S,AX,CN -6 & 1 & \\
\hline AMC,RA,S,SMZ,AX,CE -7 & $\mathbf{1}$ & \\
\hline AMC,CE,AX,S,RA,CN -8 & 1 & \\
\hline S,CFM,SMZ,AX,CE -9 & 1 & \\
\hline S,CFM,RA,ZOX,CE -10 & $\mathbf{1}$ & \\
\hline & & K.pneumoniae \\
\hline 1- 1 مقاومة لجميع المضادات & 2 & \\
\hline AK مقاومة لجميع المضادات ماعدا & 1 & \\
\hline 3- مقاومة لجميع المضادات ماعداSMZ,AK & 1 & \\
\hline 4- - مقاومة لجميع المضادات ماعداS,AK & 1 & \\
\hline 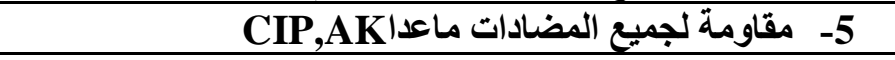 & 1 & \\
\hline AMC,CTX,CFM,CE,AX,S,RA,CN,CIP,CRO -6 & 1 & \\
\hline AMC,CE,ZOX,AX,S,SMZ,RA,CN -7 & 1 & \\
\hline & & Pr.mirabilis \\
\hline 1- مقاومة لجميع المضادات ماعدا CAZ,AK,CIP & 1 & \\
\hline SMZ,CE,CAZ,CFM,AX,ZOX -2 & 1 & \\
\hline AMC,CRO,SMZ,RA,CIP -3 & 1 & \\
\hline S,SMZ,CFM -4 & 1 & \\
\hline & & P.aeruginosa \\
\hline 1- مقاومة لجميع المضادات ماعدا AK & 2 & \\
\hline AMC,CTX,CFM,ZOX,RA,S,CE,AX,CN -2 & 1 & \\
\hline AMC,CTX,CFM,ZOX,RA,S,CE,AX -3 & 1 & \\
\hline
\end{tabular}

الا ختصارات : AMC: amoxicillin $\backslash$ clavulanate, CE:cephradine, CAZ:ceftazidime, CFM:cefixime, CRO:ceftriaxone, CTX:cefotaxime, ZOX:ceftizoxime, AK:amikacin, CN: gentamicin, S: streptomycin, RA: rifampin, CIP: ciprofloxacin, SMZ: sulfamethoxazole

تعتبر م-lactamases واحدة من الآليات الرئيسية التي تستخدمها البكتيريا لابطال التاثير التثبيطي للمضادات الحيوية التي تمتلك

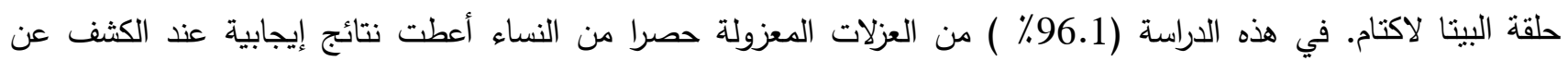
انزيمات lactamases بواسطة الطريقة الحامضية التي أشارت إلى امتلاك هذه العزلات واحد على الأقل من هذه الإنزيمات

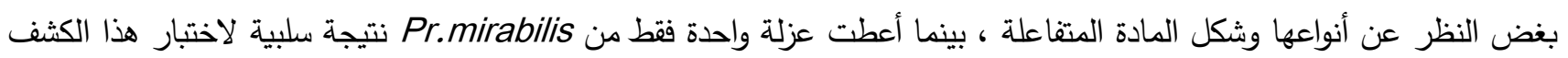

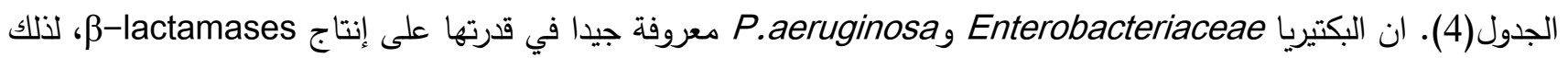

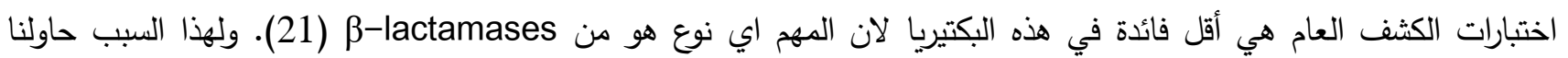
تحديد المجموعات أو الفئات التي تتتمي إليها lactamases م. ويرد في الجدول (5) ملخص لنتائج الكثف عن البيتا لاكتاميز بالتآزر المزدوج للقرص، ووجد هناك خمس عزلات فقط (19.2\%) أعطت نتائج إيجابية لهذا الاختبار، وكان من المتوقع أربعة منهم 
أن يكون نوع ESBLs مما لوحظ في توسيع منطقة التثبيط نحو قرص CTX-M كن Cefotaxime، وواحد آخر من نوع CAZ من ESBLs عندما كان التوسع في منطقة التثبيط نحو القرص Ceftazidime (22,21). ومن ناحية أخرى، أعطت نتائج الكشف عن انزيمات AmpC معدلات أعلى (69.2 \%)، فقد وجدت معدلات انتشار عالية في عزل

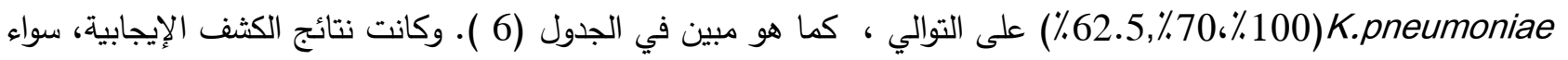

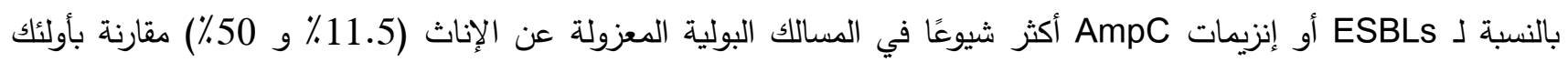

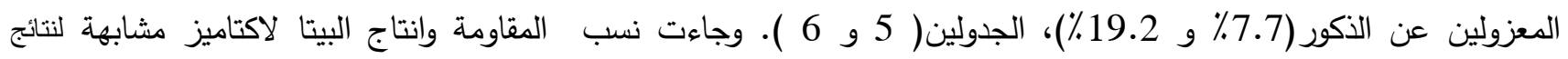
P.aeruginosa (28)Alham and.Ali الجدول (4) نتائج الكثف عن انزيم البيتا لاكتاميز بواسطة الطريقة الحامضية

\begin{tabular}{|c|c|c|c|c|}
\hline والموجبةتلالاختباري & المئويةللانكور & المئويةلكلاناث & عددعزلات & الانواع الجرثومية \\
\hline $\begin{array}{c}10(100) \\
8(100) \\
3(75) \\
4(100) \\
25(96.1)\end{array}$ & $\begin{array}{c}6(60) \\
0(0.0) \\
2(50) \\
0(0.0) \\
8(30.8)\end{array}$ & $\begin{array}{c}4(40) \\
8(100) \\
1(25) \\
4(100) \\
17(65.3)\end{array}$ & $\begin{array}{c}10 \\
8 \\
4 \\
4 \\
26\end{array}$ & $\begin{array}{c}\text { E.coli } \\
\text { K.pneumoniae } \\
\text { Pr.mirabilis } \\
\text { P.aeruginosa } \\
\text { العدد الكلي }\end{array}$ \\
\hline
\end{tabular}

الجدول(5) نتائج التحري عن انزيم البيتا لاكتاميز (ESBLs) بطريقة تازر القرص المزدوج

\begin{tabular}{|c|c|c|c|c|c|}
\hline النوع المتوقع & الموجبة والنسبة العزلات & اللمائوية & المئوية للاناث & عزلات & الانواع الجرثومية \\
\hline CTX-M & 1(10) & 1(10) & $0(0.0)$ & 10 & E.coli \\
\hline CTX-M & $2(25)$ & $\mathbf{0}(\mathbf{0 . 0})$ & $2(25)$ & 8 & K.pneumoniae \\
\hline CTX-M & $1(25)$ & $1(25)$ & $0(0.0)$ & 4 & Pr.mirabilis \\
\hline CAZ & $1(25)$ & $\mathbf{0}(\mathbf{0 . 0})$ & $1(25)$ & 4 & P.aeruginosa \\
\hline & $5(19.2)$ & $2(7.7)$ & $3(11.5)$ & 26 & العدد الكلي \\
\hline
\end{tabular}

الجدول(6) نتائج التحري عن انزيمات AMPC البيتا لاكتاميز في العزلات الجرثومية

\begin{tabular}{|c|c|c|c|c|}
\hline للعزلات الموجبة للاختبار & العدئ والنسبة للنكور & العدئ والنسبة للإناث & عدد عزلات & الانواع الجرثومية \\
\hline $7(70)$ & $4(40)$ & $3(30)$ & 10 & E.coli \\
\hline $5(62.5)$ & $0(0.0)$ & $5(62.5)$ & 8 & K.pneumoniae \\
\hline $2(50)$ & 1(25) & $1(25)$ & 4 & Pr.mirabilis \\
\hline $4(100)$ & $0(0.0)$ & $4(100)$ & 4 & P.aeruginosa \\
\hline $18(69.2)$ & $5(19.2)$ & $13(50)$ & 26 & العدد الكلي \\
\hline
\end{tabular}


ومن الجدير بالذكر أن نتائج الكثف عن AmpC هي نتائج محتملة لانتاج هذه الإنزيمات، مما يعني أنها ليست قاطعة وتحتاج إلى طرق كثف أكثر تحديدا ودقة لتكون نهائية (28,12)، ولكن لا تزال تشير إلى احتمال عالي لوجود هذه الإنزيمات في العزلات المختبرية. وهذا قد يفسر ارتفاع معدلات المقاومة التي تظهر من قبل هذه العزلات ضد المضادادات الحيوية البتا لاكتام المستخدمة

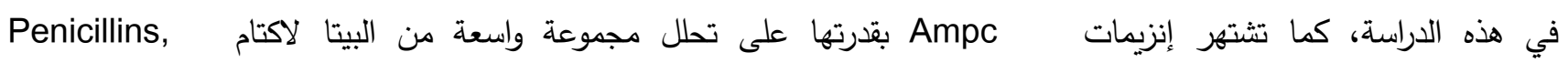
Carbapenems باستثناء Cephamycins, Monobactams,1st, 2nd and 3rd generation Cephalosporins تهر (12,15). على الرغم من أن هذه الإنزيمات عادة ما تكون كروموسومية وان تعبيرها تحت السيطرة الحثية، الا انه من المتوقع ان تشفر هذه الإنزيمات بوساطة البلازميد من خلال نقل الجينات الكروموسومية إلى البلازميدات (12,9,1)

من المهم التأكيد على أن المقاومة المتعددة للبكتريا Enterobacteriaceae و قد.aeruginosa قد يكون بسبب امتلاكها أكثر من نوع واحد من البيتا لاكتاميز التي لاتثبط بواسطة مثبطات البيتا لاكتاميز التقليدية مثل clavulanic acid حيث يكمل أحد الإنزيمات عمل الآنزيمات الاخرة وهذا قد يفسر انخفاض معدل الكثف عن ESBLs (

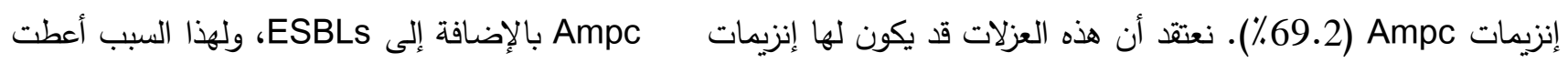

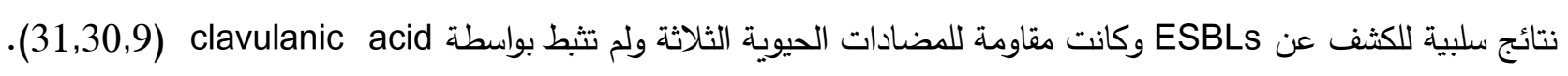
من الواضح من النتائج الواردة في هذه الدراسة أن هناك مستوى عالي من المقاومة المتعددة للأدوية بين البكتريا المعزولة من

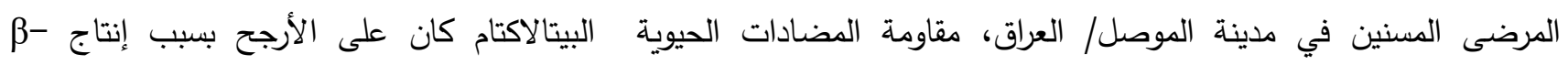

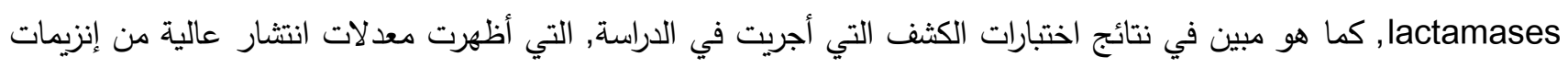
Ampc وكذلك ESBLs. الاستخدام العشوائي وبدون وصفات طبية للمضادات الميكروبات هو بالتأكيد أحد الأسباب الرئيسية

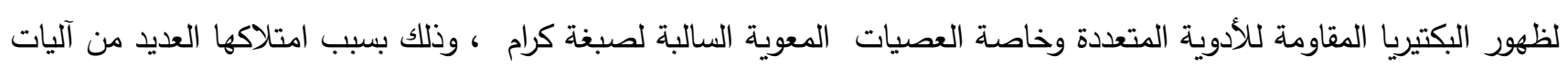

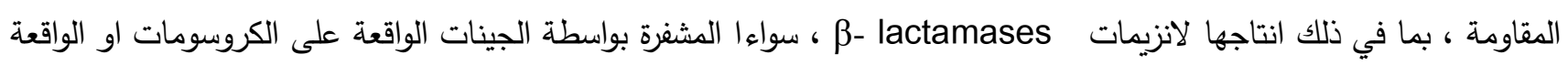

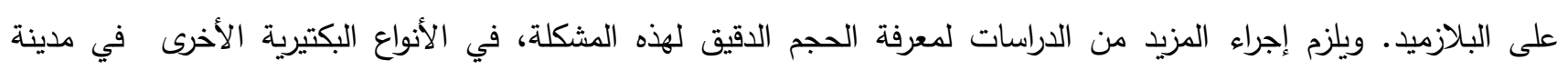
الموصل. الاستنتاجات

من المثير للقلق أن نلاحظ تقرببا أن جميع العزلات المدرجة في هذه الدراسة وجدت مقاومة لأربعة أو أكثر من المضادات

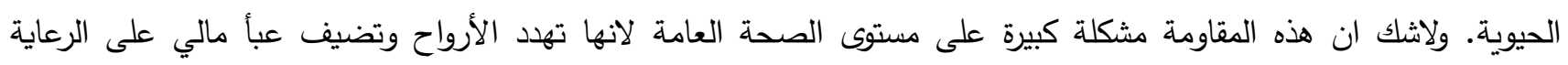

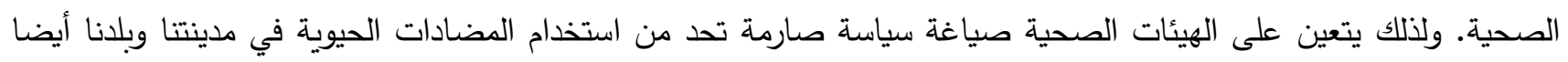
من أجل السيطرة على مشكلة مقاومة المضادات الحيوية والحد من انتشارها ومنع ظهور بكتيريا جديدة مقاومة للأدوية المتعددة.

[1] W.E. Stamm and R.S. Norrby "Urinary tract infections: disease panorama and challenges". J.Infect.Dis.,183 Suppl 1:S1-4. (2001).

[2] J.A.Karlowsky , M.E. Jones and C.Thrnsberry "Prevalence of antimicrobial resistance among urinary tract pathogens isolated from female outpatients across the US in 1999”.Int.Antimicrob.Agents, 18:121-127. (1999). 
[3] H.Arslan , O.K. Azap, O.Ergonul and F. Timurkaynak "Risk factors for ciprofloxacin resistance among Escherichia coli strains isolated from community-acquired urinary tract infections in Turkey”. J.Antimicrob.Chemother.,56:914-918.(2018)

[4] M.Akram , M.Shahid and A.Khan, "Etiology and antibiotic resistance patterns of communityacquired urinary tract infections in JNMC hospital Aligarh, India". Ann. Clin. Microbiol. Antimicrob., 6:4-10. (2007).

[5] E.W. Koneman , S.D. Allen , W.M. Janda , P.C. Schreckenberger and W. C.Winn "Color Atlas and Textbook of Diagnostic Microbiology" . $5^{\text {th }}$ ed., Lippincott-Raven publisher ,Philadelphia, U.S.A. (1997).

[6] D.M. Livermore, " $\beta$-lactamases in laboratory and clinical resistance" . Clin.Microbiol.Rev.,8: 557-584(1995).

[7] K.Bush , G.A. Jacoby and A.A. Medeiros "A functional classification scheme for $\beta$-lactamases and its correlation with molecular structure" . Antimicrob. Agents Chemother., 39: 1211-1233 (1995).

[8] P.Bradford "AExtended-spectrum $\beta$-lactamases in the $21^{\text {st }}$ century: characterization, epidemiology, and detection of this important resistant threat" . Clin.Microbiol.Rev.,14: 933951.(2001).

[9] K.S. Thomson "Controversies about extended-spectrum and AmpC $\beta$-lactamases" . Emerg.Infect.Dis., 7: 333-336(2001).

[10] M.A. Pfaller and J.Segreti "Overview of the epidemiological profile and laboratory detection of extended-spectrum $\beta$-lactamases”. Clin.Infect.Dis.,42 Suppl 4:S153-162. (2006).

[11] J.R. Knox "Extended-spectrum and inhibitor-resistant TEM-type $\beta$-lactamases: Mutations, specificity, and three-dimensional structure" . Antimicrob. Agent Chemother., 39: 2593-2601(1995).

[12] K.Nasim , , S.Elsayed , J. D. D.Pitout , J.Conly , D. L.Church and D.B. Gregson "New method for laboratory detection of AmpC $\beta$-lactamases in Escherichia coli and Klebsiella pneumonia" . J.Clin.Microbiol., 42: 4799-4802(2004).

[13] V.Manchanda and N.P. Singh "Occurrence and detection of AmpC $\beta$-lactamases among gramnegative clinical isolates using a modified three-dimensional test at Guru Tegh Bahadur hospital ,Delhi, India” .J.Antimicrob.Chemother., 51: 415-418(2003).

[14] A.M.Queenan, S.Jenkins and K.Bush "Cloning and biochemical characterization of FOX-5, an AmpC-type plasmid-encoded $\beta$-lactamase from a New York city Klebsiella pneumoniae clinical isolate" . Antimicrob. Agents Chemother., 45: 3189-3194(2001).

[15] A.Philippon , G.Arlet and G.A. Jacoby "Plasmid AmpC-type $\beta$-lactamases" . Antimicrob. Agents Chemother., 46: 1-11(2002).

[16] E.J.Baron , L.R.Peterson, and S.M.Finegold "Bailey\&Scott's Diagnostic Microbiology" . $9^{\text {th }}$. ed.,Mosby-Year Book ,Inc.,U.S.A. .(1994)

[17] J.F. MacFaddin "Biochemical Tests for Identification of Medical Bacteria" . 2 $2^{\text {nd }}$ ed., Williams\&Wilkins company , Baltimore , U.S.A. . (1985) 
[18] National Committee for Clinical Laboratory Standards, NCCLS "Performance Standards for Antimicrobial Disk Susceptibility Tests" ;Approved Standards- $8^{\text {th }}$ ed., M2-A8, Vol.20 , No.1 ,NCCLS , Wayne, PA, U.S.A. (2003).

[19] A.E.Brown "Benson`s Microbiological Applications Laboratory Manual in General Microbiology”10thed., P. 102-263. McGraw-Hill comp. Inc.,

USA. .(2007)

[20] National Committee for Clinical Laboratory Standards, NCCLS "Performance Standards for Antimicrobial Susceptibility Testing ; $14^{\text {th }}$ Informational $\quad$ Supplement" $\quad$ M100S14 ,Vol.24 ,No.1 ,NCCLS, Wayne , PA , U.S.A. (2004).

[21] D.M.Livermore, and D.F.J.Brown "Detection of $\beta$-lactamase-mediated resistance". J. Antimicrob. Chemother., 48 Suppl 1:S59-64.(2001)

[22] C.C. Sanders , A.L. Barry , J.A. Washington , C.Shubert , E.S.Moland , M.M. Traczewski , C.Knapp , and R.Mulder, "Detection of extended-spectrum- $\beta$-lactamase-producing members of the family Enterobacteriaceae with the Vitek ESBL test”. J.Clin.Microbiol., 34: 2997-3001(1996).

[23] P.E.Coudronm, E.S. Moland, and K.S.Thomson "Occurrence and detection of AmpC $\beta$ lactamases among Escherichia coli, Klebsiella pneumoniae, and Proteus mirabilis isolates at a veterans medical center". J.Clin.Microbiol.,38: 1791-1796 .(2000).

[24] T.M. File "Overview of resistance in the 1990s" .Chest 115 Suppl:3S-8. .(1999)

[25] L.M. Prescott , J.P. Harley and D.A. Klein “ Microbiology” . $3^{\text {rd }}$ ed., Wm.C. Brown Communication, Inc., Iowa, U.S.A. (1996).

[26] M.Z.Al-Hasso "Extraction and purification of $\beta$-lactamases from some Gram negative bacilli isolated from lower respiratory tract infections and study of some of their characteristics". Ph.D thesis, College of Science, Mosul University.(in Arabic) (2006).

[27] N.D. Hanson "AmpC $\beta$-lactamases : What do we need to know for the future ?" . J.Antimicrob.Chemother.,52:2-4(2003).

[28] J.K.Alham and M.Ali "Investigation of broad-spectrum bitachemic enzymes in clinical isolation Pseudomonas aeruginosa in Najaf” AL Kufa University Journal for Bilogy Vol 4 No 1 (2012).

[29] M.Stoczko , J. Frere, G.Rossolini and J.Docquier "Antimicro". Agents

Chemother.,11: 1973-1981. (5) Livermore, D. M., Clin. Microbiol. Rev.,8: 5(2006).

[30] J. N . Samaha-Kfouryand , G. F.Araj BM J, 327:1209-1213 (2003) .

[31] M.A. Mahmood , M.A. Essa "Antimicrobial activity of peptides extracted from camels' blood neutrophils against some pathogenic bacteria" Iraqi Journal of Veterinary Sciences, Vol. 35, No. 1,33-37 (2021) . 